\title{
Telomere Length and Risk of Hepatocellular Carcinoma: A Nested Case-control Study in Taiwan Cancer Screening Program Cohort
}

\author{
HUI ZENG ${ }^{1,2}$, HUI-CHEN WU ${ }^{1}$, QIAO WANG ${ }^{1}$, HWAI-I YANG ${ }^{3,4,5}$, \\ CHIEN-JEN CHEN ${ }^{5,6}$, REGINA M. SANTELLA ${ }^{1}$ and JING SHEN ${ }^{1}$ \\ ${ }^{1}$ Department of Environmental Health Sciences, Mailman School of Public Health, \\ Columbia University Medical Center, New York City, NY, U.S.A.; \\ ${ }^{2}$ Department of Environmental Hygiene, College of Preventive Medicine, \\ Third Military Medical University, Chongqing, P.R. China; \\ ${ }^{3}$ Graduate Institute of Clinical Medical Science, China Medical University, Taichung, Taiwan, R.O.C.; \\ ${ }^{4}$ Molecular and Genomic Epidemiology Center, China Medical University Hospital, Taichung, Taiwan, R.O.C.; \\ ${ }^{5}$ Genomics Research Center, Academia Sinica, Taipei, Taiwan, R.O.C.; \\ ${ }^{6}$ Graduate Institute of Epidemiology and Preventive Medicine, \\ College of Public Health, National Taiwan University, Taipei, Taiwan, R.O.C.
}

\begin{abstract}
Background: Telomere length (TL) measured in peripheral blood leucocytes (PBL) might be a useful biomarker to identify elevated cancer risk. Patients and Methods: A casecontrol study which included 268 newly-diagnosed HCC cases and 536 matched controls, was conducted. Absolute TL in PBL was analyzed by quantitative real-time PCR. Results: The overall median length of TL was not statistically shorter in HCC cases compared to healthy controls. However, we found a significant synergistic effect of longer TL and HCV infection to increase HCC risk with a relative excess risk of $6.86(95 \%$ CI: 2.14-11.58). Among HCC cases, significant shorter TLs were observed for $<5$ years $(O R=3.93,95 \%$ CI: 2.00-7.72); 5 10 years $(O R=2.16,95 \% C I: 1.10-4.24)$ compared to $>10$ years prior to diagnosis. Conclusion: Shorter PBL TL alone was not significantly associated with increased HCC risk. Among HCC cases, significant shorter TLs were observed for $<5$ years prior to diagnosis.
\end{abstract}

Telomeres are tandem repeats of TTAGGG nucleotides located at the end of chromosomes and are essential to prevent chromosomal fusions and maintain genomic stability

Correspondence to: Jing Shen, Ph.D., Department of Environmental Health Sciences, Mailman School of Public Health, Columbia University Medical Center, 650 West 168th St New York NY 10032, U.S.A. Tel: +1 2123058158, e-mail: js2182@ cumc.columbia.edu

Key Words: HCC, telomere length, nested case-control study, quantitative PCR.
(1). Telomere length (TL) is shortened 50 to 200 nucleotides per cell division because of the "end-replication problem" ( 2 , 3 ). Telomerase, a ribonucleic protein, can add telomeric sequences onto chromosome ends to maintain the length of telomere. Thus TL is dynamic with shortening and lengthening both taking place. In physiological cells, telomere shortening acts as a mitotic clock and can signal replicative senescence to cells in order to become senescent or apoptotic. However, when telomere shortening reaches a critical stage, it may cause a catastrophic event increasing the risk of cancer initiation (4) because of loss of telomere capping function and chromosomal stability during replication (5). Furthermore, in the majority of cancer cells the shortened telomeres are elongated by telomerase activation allowing cells to replicate and maintaining tumor growth.

Hepatocellular carcinoma (HCC) is one of the most common human malignancies. The incidence rate is more than 20 per 100,000 in sub-Saharan Africa and in Eastern Asia. Most prior studies, including ours (6), reported that TL is significantly shortened in HCC tumor tissues compared to surrounding non-tumor tissue (7). The shortening of TL plays a dual role in hepatocarcinogenesis, (7) i.e. accelerating tumor initiation and suppressing tumor progression because the chromosomal instability activates the DNA-damage response and apoptosis pathways in tumor cells (7). Two human studies showed that telomere shortening occurred in pre-neoplastic lesions of dysplastic nodules during multistep hepatocarcinogenesis, indicating that telomere shortening is an early tumorigenic event $(8,9)$. However, Oh et al. reported that $55.6 \%$ of $\mathrm{HCC}$ tumor 
tissues had shorter telomeres and $44.4 \%$ had longer telomeres than adjacent non-neoplastic tissues (10).

Because it is relatively easier to obtain peripheral blood leukocytes (PBLs) than cancer tissues, TL measured in leukocytes has been used as a surrogate of target tissue to explore the association with cancer risk and prognosis. Shorter TL in leukocytes is associated with increased risk of lung and oropharyngeal squamous cell carcinoma as well as renal, gastric and esophageal cancers, but TL association with liver cancer remains unclear. One case-control study showed that longer TL in leukocytes was associated with increased risk of HBV-related HCC after adjusting for age, gender, smoking and alcohol drinking. A significant dose-response relationship was also observed (11). This study was limited by its retrospective case-control study design that measured TL in PBLs after cancer diagnosis. Thus it is unclear whether the longer TL was a risk factor or a consequence of tumorigenesis. Another hospital-based nested case-control study measured serum DNA TL, which represents the TL in a variety of cells (white blood cells, dead cells and circulating tumor cells). Longer TL was significantly associated with risk of HBV-related HCC in univariate analysis, but became not significant after adjusting for age, gender, smoking, alcohol drinking, cirrhosis and family history of cancer (12). Whether TL measured in PBLs collected prior to HCC diagnosis is associated with $\mathrm{HCC}$ risk or related to the common risk factors of HCC is largely unknown. Here, we conducted a case-control study nested in the Taiwan Cancer Screening Program (CSP) cohort to prospectively examine the association between TL and HCC risk.

\section{Patients and Methods}

Study cohort and participants. The CSP cohort is a prospective study that was originally set up to evaluate the efficacy of cancer screening in Taiwan. The characteristics of this cohort have been described previously (13). Briefly, a total of 12,020 males and 11,923 females between 30 and 64 years old were enrolled in the cohort from July 1990 to June 1992 in seven urban townships with high HCC incidence rates. Epidemiologic information including sociodemographic characteristics, habits of alcohol intake, cigarette smoking and HCC family history were obtained using a structured questionnaire based on personal interview. Habitual cigarette smoking was defined as having smoked more than 4 days a week for at least 6 months. Habitual alcohol intake was defined as drinking alcohol-containing products more than 4 days a week for at least 6 months. Participants also donated a $20 \mathrm{~mL}$ fasting blood sample when recruited. Samples were transported to a central laboratory at National Taiwan University, and PBLs, plasma and red blood cell were separated and stored at $-70^{\circ} \mathrm{C}$. $\mathrm{HBV}$ surface antigen (HBsAg) was tested by radioimmunoassay (Abbott Laboratories, Taipei, Taiwan); antibodies against HCV (anti-HCV) and alpha-fetoprotein (AFP) levels were tested by enzyme immunoassay (Abbott Laboratories, Taipei, Taiwan). This study was approved by Columbia University's Institutional Review Board and the Research Ethics
Committee of the College of Public Health, National Taiwan University. Written informed consent was obtained from all subjects.

The criteria for HCC diagnosis included a histopathologic confirmation or positive lesion detected by at least two different imaging techniques (abdominal ultrasonography, angiogram, or computed tomography) or by one imaging technique and a serum AFP level of $>400 \mathrm{ng} / \mathrm{mL}$. Participants who had an elevated level of alanine transaminase $\geq 45 \mathrm{IU} / \mathrm{mL}$ ), aspartate transaminase ( $\geq 40 \mathrm{IU} / \mathrm{mL}$ ), or AFP ( $\geq 20 \mathrm{ng} / \mathrm{mL}$ ), were positive for HBsAg or anti-HCV, or had a family history of HCC or liver cirrhosis among first-degree relatives were referred for upper abdominal ultrasonographic examination. Participants with ultrasonographic images indicative of liver cirrhosis had intensive follow-up, including abdominal ultrasonographic screening and serum AFP level determination every 3 month. Suspected HCC cases were confirmed by computerized tomography, digital subtracted angiogram, aspiration cytology, and pathologic examination.

Other participants of this cohort were passively followed up and new HCC cases were identified by linking with the national cancer registry and death certification systems in Taiwan. In the current study, a total of $268 \mathrm{HCC}$ cases were newly diagnosed during the time of follow-up from February 1991 to June 2004 and also had PBL DNA for TL measurement. We then randomly selected from all participants who were not affected with HCC through the followup period and matched 2 controls with each case by age ( \pm 5 years), gender, residential township and date of recruitment ( \pm 3 months). Finally, 536 controls were selected for the current study that also had PBL DNA for TL measurement.

Absolute telomere length measurement. Telomere length (TL) was determined by modified single well multiplex quantitative PCR (1) combined with the estimation of absolute TL as described previously (2). First, the total TL per reaction (sample) was determined by the quantification cycle $\left(\mathrm{C}_{\mathrm{q}}\right)$ of telomeres using a standard curve generated from serial dilution of a synthesized telomeric oligonucleotide. The genome copy numbers per reaction (sample) were determined by the $\mathrm{C}_{\mathrm{q}}$ of albumin using a standard curve generated from serial dilution of a synthesized albumin oligonucleotide. Then, the absolute TL $(\mathrm{kb})$ per human diploid genome was calculated using the total TL per reaction divided by the diploid genome copies of albumin per reaction.

The standard curve for TL was generated using a synthesized 84 bp oligonucleotide containing 14 TTAGGG repeats (Invitrogen, New York City, NY, USA) with a molecular weight (MW) of 25113. The weight of one molecule of the telomere standard is equal to MW divided by Avogadro's number $\left(25113 / 6.02 \times 10^{23}=4.17 \times 10^{-20} \mathrm{~g}\right)$. The highest concentration in the standard curve (TEL STD A) was $1 \mathrm{pg}$ of telomere oligomer $\left(1 \times 10^{-12} \mathrm{~g}\right)$ per reaction $(15 \mu \mathrm{l})$, and there are $1 \times 10^{-12} / 4.17 \times 10^{-20}=2.4 \times 107$ molecules of oligomer in TEL STD A. The standard curve is generated from qPCR analysis of 5 serial dilutions of TEL STD (from $2.4 \times 10^{7}$ to $1.5 \times 10^{6}$ molecules). So, the length of telomere sequence in TEL STD A is calculated as: $2.4 \times 10^{7} \times 84$ (oligomer length) $=2.02 \times 10^{6} \mathrm{~kb}$.

The albumin standard curve was generated using a synthesized 44 mer oligonucleotide (AAATGCTGCACAGAATCCTTGGTGA ACAGGCGACCATGCTTTTC) that has a MW of 13516 (Invitrogen) to estimate genome copy number per reaction. The weight of one molecule of albumin oligonucleotide is equal to $13516 / 6.02 \times 10^{23}=2.25 \times 10^{-20} \mathrm{~g}$. The highest concentration in the standard curve (Alb STD A) was $0.2 \mathrm{pg}$ of albumin oligomer 
$\left(0.2 \times 10^{-12} \mathrm{~g}\right)$ per reaction, or $0.2 \times 10^{-12} / 2.25 \times 10^{-20}=8.9 \times 10^{6}$ copies of the albumin amplicon in Alb STD A. Because there are two copies of albumin per diploid genome, the Alb STD A is equivalent to $4.45 \times 10^{6}$ diploid genome copies.

The PCR primers were synthesized (Life Science) according to a previous report (Cawthon, 2009). The telomere primers telg (ACACTAAGGTTTGGGTTTGGGTTTGGGTTTGGGTTAGTGT) and telc (TGTTAGGTATCCCTATCCCTATCCCTATCCCTATCCC TAACA) can generate a short, fixed-length product (76 bp). Only telg is able to prime DNA synthesis along native telomeric DNA sequences, and the telc primer cannot prime DNA synthesis due to its 3 ' terminal mismatch. The albumin primers albu (CGGCGGCGG GCGGCGCGGGCTGGGCGGaaatgctgcacagaatcettg) and albd (GCCCGGCCCGCCGCGCCCGTCCCGCCGgaaaagcatggtcg cctgtt) can generate a product of $98 \mathrm{bp}$. Non-templated 5' ends (capitalized bases) are used to ensure the $\mathrm{T}_{\mathrm{m}}$ value of the albumin PCR product $\left(88^{\circ} \mathrm{C}\right)$ is higher than the telomere product $\left(74^{\circ} \mathrm{C}\right)$. SYBR Green I fluorescence signals collected at $88^{\circ} \mathrm{C}$ only indicate the amount of albumin amplicons, but the signals collected at $74^{\circ} \mathrm{C}$ represent both albumin and telomere amplicons. Because the copy numbers of albumin are much lower than the telomere template, the signals from albumin at $74^{\circ} \mathrm{C}$ can be neglected compared to the signals for telomeres for each DNA sample. Thus, both telomere and albumin quantification were conducted within the same well to minimize the variations of input DNA amount and PCR efficiency.

The final volume of each quantitative PCR was $15 \mu$ l containing approximately $40 \mathrm{ng}$ of DNA and was run in a Bio-Rad iQ5 realtime PCR detection system. Five concentrations of synthesized oligonucleotides for telomere and albumin were prepared by serial dilution and analyzed in duplicate for each 96 -well plate. These data were used to generate standard curves for unknown sample quantitation. Each DNA sample was assayed in duplicate. The final concentrations of reagents in the PCR were $1 \times$ SYBR Green I (Invitrogen), $10 \mathrm{mM}$ Tris- $\mathrm{HCl} \mathrm{pH} 8.3,50 \mathrm{mM} \mathrm{KCl}, 3 \mathrm{mM} \mathrm{MgCl}{ }_{2}$, $0.2 \mathrm{mM}$ each dNTP, $1 \mathrm{mM}$ DTT. Each $15 \mu \mathrm{l}$ reaction received $1.5 \mathrm{U}$ AmpliTaq Gold DNApolymerase (Applied Biosystems, Inc. New York City, NY, USA). Reactions were run with telomere primers (telg/telc) at $360 \mathrm{nM}$ each and albumin (single copy gene) primers (albu/albd) at $90 \mathrm{nM}$ each. The thermal cycling profile was $15 \mathrm{~min}$ at $95^{\circ} \mathrm{C}, 2$ cycles of $15 \mathrm{~s}$ at $94^{\circ} \mathrm{C}, 15 \mathrm{~s}$ at $49^{\circ} \mathrm{C}$, and 32 cycles of $15 \mathrm{~s}$ at $94^{\circ} \mathrm{C}, 10 \mathrm{~s}$ at $62^{\circ} \mathrm{C}$, and $15 \mathrm{~s}$ at $74^{\circ} \mathrm{C}$ with signal acquisition, $10 \mathrm{~s}$ at $84^{\circ} \mathrm{C}$, and $15 \mathrm{~s}$ at $88^{\circ} \mathrm{C}$ with signal acquisition. The $74^{\circ} \mathrm{C}$ reads provided the $\mathrm{C}_{\mathrm{q}}$ values for the amplification of the telomere template (the albumin signal is still at baseline); the $88^{\circ} \mathrm{C}$ reads provided the $\mathrm{C}_{\mathrm{q}}$ values for the amplification of albumin template (there is no signal from the telomere PCR product because it is fully melted).

To evaluate the reproducibility of the assay, $5 \%$ of randomly selected samples were assayed again on different plates and the average coefficient of variation (CV) was 0.28 for absolute TLs measurement. When categorizing TLs into shorter $(<42.19 \mathrm{~kb} /$ genome $)$ or longer ( $\geq 42.19 \mathrm{~kb} /$ genome) according to the median in controls, the observed the agreement for the 5\% repeated samples was $86 \%$ (Kappa coefficient=0.72), indicating the strength of agreement to be 'substantial'.

Statistical analysis. The distribution of participants' characteristics between cases and controls were evaluated by Pearson Chi-square test. TL was analyzed as both a continuous and categorical variable. The Wilcoxon rank sum test was used to evaluate the difference in TL as a continuous variable by case-control status. In the initial data analysis, median, tertile and quartile values of TL in the control group were used as cutoff points. The association between TL and HCC risk was separately assessed using conditional logistic regression to determine odds ratios (ORs) and 95\% confidence intervals (CIs). The associations between blood collection prior to HCC diagnosis ( $<5$ years vs. 5-10 years vs. $>10$ years) and TLs were assessed to determine ORs and 95\% CIs. Oneway analysis of variance (ANOVA) was used to determine the differences of TLs by blood collection groups. The potential synergetic or antagonistic interaction effect (i.e. modification effect) between TLs and HBV/HCV infection, cigarette smoking and alcohol drinking were analyzed by conditional logistic regression models, and the relative excess risk (RER) due to interaction was estimated to evaluate the magnitude of the additive effect according to Andersson's method (14), i.e. $\mathrm{RER}=\mathrm{OR}_{(\mathrm{AB})^{-}}$ $\mathrm{OR}_{(\mathrm{A})}-\mathrm{OR}_{(\mathrm{B})}+1 . \mathrm{OR}_{(\mathrm{AB})}$ is the relative risk for co-exposure to factor $\mathrm{A}$ and $\mathrm{B} ; \mathrm{OR}_{(\mathrm{A})}$ is the relative risk for only exposure to factor $A$, while $\mathrm{OR}_{(\mathrm{B})}$ is the relative risk for only exposure to factor $B$. RER equal to 0 indicates no biological interaction, while RER $>0$ or RER $<0$ suggesting a potentially synergetic or antagonistic interaction, respectively. All statistical analyses were conducted using SPSS 20.0 and $p$-values less than 0.05 were considered as statistically significant (2-sided).

\section{Results}

The sociodemographic characteristics of HCC cases and controls are shown in Table I. No statistically significant differences were observed for age, gender, BMI and cigarette smoking by case-control status. Significantly higher frequencies were found among $\mathrm{HCC}$ cases compared to controls for HBsAg-positive (63.4\% vs. $25.6 \%)$, anti-HCVpositive $(25.1 \%$ vs. $9.6 \%)$ and alcohol drinking cases $(17.6 \%$ vs. $12.0 \%)$.

The final available data for TL were 801 (268 HCC cases and 533 controls) with only 3 missing. The overall median levels of TL were not statistically different between cases and controls (38.98vs. $42.19 \mathrm{~kb} /$ genome, $p=0.24$, Table II). When stratified by age, gender, BMI, HBsAg, anti-HCV, and smoking status, TL was also not significantly different between cases and controls. The only case-control difference was observed among ever-alcohol-drinkers whose TL was significantly shorter in cases $(27.62 \mathrm{~kb} /$ genome $)$ compared to controls ( $36.96 \mathrm{~kb} / \mathrm{genome}, p=0.01)$. This difference is entirely due to males, because there were no female alcohol drinkers (data not shown). Table II also shows that TL was significantly shorter in males than females in both, test cases ( 35.21 vs. $55.13 \mathrm{~kb} / \mathrm{genome}, p<0.0001)$ and controls (36.56 vs. $53.08 \mathrm{~kb} /$ genome, $p<0.0001)$. Significantly shorter TL was observed for HBsAg positive vs. negative subjects and ever smokers $v s$. never smokers in both, cases and controls. Shorter TL was observed in ever alcohol drinkers compared to non-drinkers but was only significant in cases $(27.62 \mathrm{vs}$. $41.88 \mathrm{~kb} /$ genome, $p=0.001)$. In controls, TL was significantly shorter in anti-HCV positive compared to antiHCV-negative (34.01 vs. $43.96 \mathrm{~kb} /$ genome, $p=0.04$ ) subjects. 
Table I. Sociodemographic Characteristics of HCC Cases and controls

\begin{tabular}{|c|c|c|c|}
\hline Variables & Cases $(\mathrm{N}=268)$ & Controls $(\mathrm{N}=536)$ & $p$-Value \\
\hline Age (Years \pm SD) & $53.48 \pm 7.62$ & $53.39 \pm 7.68$ & $0.88 *$ \\
\hline \multirow[t]{2}{*}{ BMI (Mean \pm SD) } & $24.54 \pm 3.87$ & $24.63 \pm 4.15$ & $0.77 *$ \\
\hline & $\mathrm{N}(\%)$ & $\mathrm{N}(\%)$ & \\
\hline \multicolumn{4}{|l|}{ By median of BMI } \\
\hline$<25$ & $153(57.1)$ & $308(57.5)$ & 0.92 \\
\hline$\geq 25$ & $115(42.9)$ & $228(42.5)$ & \\
\hline \multicolumn{4}{|l|}{ Gender } \\
\hline Female & $89(33.2)$ & $178(33.2)$ & 1.00 \\
\hline Male & $179(66.8)$ & $358(66.8)$ & \\
\hline \multicolumn{4}{|l|}{$\mathrm{HBsAg}$} \\
\hline- & 97 (36.6) & $396(74.4)$ & $<0.001$ \\
\hline+ & $168(63.4)$ & $136(25.6)$ & \\
\hline \multicolumn{4}{|l|}{ Anti-HCV } \\
\hline- & 179 (74.9) & $422(90.4)$ & $<0.001$ \\
\hline+ & $60(25.1)$ & $45(9.6)$ & \\
\hline \multicolumn{4}{|l|}{ Cigarette smoking } \\
\hline Never & $163(60.8)$ & $335(62.6)$ & 0.61 \\
\hline Ever & $105(39.2)$ & $200(37.4)$ & \\
\hline \multicolumn{4}{|l|}{ Alcohol drinking } \\
\hline Never & $220(82.4)$ & $471(88.0)$ & 0.04 \\
\hline Ever & $47(17.6)$ & $64(12.0)$ & \\
\hline
\end{tabular}

*The Wilcoxon rank sum test for continuous variable.

We performed a conditional logistic regression analysis to assess the association between shorter TL and HCC risk (Table III). When participants were categorized according to the median, tertile or quartile values of TL in healthy controls, shorter TL was not associated with increased risk of HCC. When stratified by HBV or HCV infection, or cigarette smoking and alcohol drinking status, shorter TL was not associated with a significant increase in HCC risk (data not shown).

The potential modification effects between TL and HCC risk factors (positive for $\mathrm{HBsAg}$, anti-HCV, ever smoking and ever alcohol drinking) were also analyzed (Table IV). No significant modification effect was observed between TL and HBsAg status and ever cigarette smoking. However, anti-HCV positive subjects with longer TL was associated with a significant modification effect to increase HCC risk (OR=11.47, 95\% CI: 4.10-32.08) even after adjusting for age, gender and other HCC risk factors. Ever alcohol drinkers with shorter TL also associated with a significant modification effect to increase $\mathrm{HCC}$ risk $(\mathrm{OR}=2.27,95 \%$ CI: 1.25-4.13), but the significance disappeared after adjusting for age, gender and other risk factors $(\mathrm{OR}=1.96$, 95\% CI: 0.92-4.19).

We analyzed TL with respect to differences in time between blood collection and diagnosis (Table V) and
Table II. Comparisons of medians of peripheral blood leucocyte telomere length ( $\mathrm{kb} /$ genome) and IQR by demographic characteristics in HCC cases and controls.

\begin{tabular}{|c|c|c|c|}
\hline Variables & Cases & Controls & $p$-Value* \\
\hline TL, Median (IQR) & $38.98(44.17)$ & $42.19(44.23)$ & 0.24 \\
\hline \multicolumn{4}{|l|}{ Age (years) } \\
\hline$<60$ & $38.58(44.92)$ & $44.10(43.56)$ & 0.12 \\
\hline$\geq 60$ & $40.21(36.53)$ & $37.73(43.88)$ & 0.71 \\
\hline$p^{*}$ & 0.75 & 0.19 & \\
\hline \multicolumn{4}{|l|}{ BMI } \\
\hline$<25$ & 39.99 (39.93) & $41.69(47.37)$ & 0.51 \\
\hline$\geq 25$ & $35.65(48.39)$ & $42.44(39.36)$ & 0.26 \\
\hline$p^{*}$ & 0.80 & 0.69 & \\
\hline \multicolumn{4}{|l|}{ Gender } \\
\hline Female & $55.13(49.47)$ & $53.08(42.49)$ & 0.89 \\
\hline Male & $35.21(35.11)$ & $36.56(41.97)$ & 0.20 \\
\hline$p^{*}$ & $<0.0001$ & $<0.0001$ & \\
\hline \multicolumn{4}{|l|}{ HBsAg } \\
\hline- & $53.27(43.06)$ & $46.76(48.04)$ & 0.33 \\
\hline+ & $35.99(33.82)$ & $35.28(32.31)$ & 0.94 \\
\hline$p^{*}$ & $<0.0001$ & 0.001 & \\
\hline \multicolumn{4}{|l|}{ Anti-HCV } \\
\hline- & $38.12(41.84)$ & $43.96(45.14)$ & 0.06 \\
\hline+ & $42.13(40.64)$ & $34.01(23.73)$ & 0.06 \\
\hline$p^{*}$ & 0.17 & 0.04 & \\
\hline \multicolumn{4}{|l|}{ Cigarette smoking } \\
\hline Never & $44.10(42.79)$ & $46.34(44.82)$ & 0.99 \\
\hline Ever & $33.71(36.31)$ & $37.21(42.92)$ & 0.09 \\
\hline$p^{*}$ & 0.002 & 0.048 & \\
\hline \multicolumn{4}{|l|}{ Alcohol drinking } \\
\hline Never & $41.88(41.12)$ & $43.14(44.66)$ & 0.96 \\
\hline Ever & $27.62(28.83)$ & $36.96(32.23)$ & 0.01 \\
\hline$p^{*}$ & 0.001 & 0.60 & \\
\hline
\end{tabular}

IQR, interquartile range; *Wilcoxon rank sum test.

found that the median TLs were significantly shorter for blood collection in the years close to HCC diagnosis $(p<0.001)$. A higher proportion of patients with shorter TLs was observed in samples collected close to cancer diagnosis (76.5\% for $<5$ years, $54.7 \%$ for $5-10$ years and $37.0 \%$ for $>10$ years). Compared to bloods collected $>10$ years prior to diagnosis, blood samples collected close to diagnosis showed significantly increased risks of shorter TLs $(<5$ years with an OR of $3.93,95 \% \mathrm{CI}: 2.00-7.72 ; 5-10$ years with an OR of $2.16,95 \%$ CI: 1.10-4.24) after adjustment for age, sex, smoking, alcohol use, HBV infection and HCV infection. Interestingly, compared to controls, shorter TL significantly decreased $\mathrm{HCC}$ risk (OR=0.44, 95\% CI: 0.28 0.71 ) in blood collected over 10 years prior to diagnosis. In contrast, shorter TL was associated with increased risk of HCC in blood collected $<5$ years before diagnosis $(\mathrm{OR}=1.74,95 \% \mathrm{CI}: 0.97-3.12)$ although it did not reach statistical significance (data not shown). 
Table III. Peripheral blood leucocyte telomere length categorized by median, tertile and quartile and HCC risk.

\begin{tabular}{|c|c|c|c|c|}
\hline \multirow[t]{2}{*}{ TLs categorization } & \multicolumn{2}{|c|}{$\mathrm{N}(\%)$} & \multirow[t]{2}{*}{ Crude OR $(95 \% \mathrm{CI})$} & \multirow[t]{2}{*}{$\mathrm{OR} *(95 \% \mathrm{CI})$} \\
\hline & Cases & Controls & & \\
\hline \multicolumn{5}{|l|}{ By median } \\
\hline Longer $(\geq 42.19)$ & $124(46.3)$ & $266(49.9)$ & 1.00 (Reference) & 1.00 (Reference) \\
\hline Shorter $(<42.19)$ & $144(53.7)$ & $267(50.1)$ & $1.20(0.85-1.69)$ & $0.96(0.63-1.46)$ \\
\hline \multicolumn{5}{|l|}{ By tertile } \\
\hline Tertile3 $(\geq 58.65)$ & $87(32.4)$ & $177(33.2)$ & 1.00 (Reference) & 1.00 (Reference) \\
\hline Tertile2 ( $\geq 31.44$ to 58.64$)$ & $80(29.9)$ & $179(33.6)$ & $0.95(0.64-1.39)$ & $0.71(0.46-1.16)$ \\
\hline Tertile1 $(<31.44)$ & $101(37.7)$ & $177(33.2)$ & $1.27(0.82-1.96)$ & $1.11(0.65-1.91)$ \\
\hline \multicolumn{5}{|l|}{ By quartile } \\
\hline Q4 $(\geq 71.90)$ & $58(21.6)$ & $132(24.8)$ & 1.00 (Reference) & 1.00 (Reference) \\
\hline Q3 ( $\geq 42.19$ to 71.89$)$ & $66(24.6)$ & $134(25.1)$ & $1.17(0.75-1.81)$ & $1.19(0.72-1.98)$ \\
\hline Q2 ( $\geq 27.68$ to 42.18$)$ & $69(25.7)$ & $134(25.1)$ & $1.26(0.80-2.00)$ & $0.93(0.54-1.61)$ \\
\hline Q1 $(<27.68)$ & $75(28.1)$ & $133(25.0)$ & $1.48(0.88-2.47)$ & $1.37(0.73-2.59)$ \\
\hline
\end{tabular}

*OR adjusted by HBsAg, Anti-HCV status, cigarette smoking and alcohol drinking status.

Table IV. Potential interactions between peripheral blood leucocyte telomere length (TL) and HCC risk factors.

\begin{tabular}{|c|c|c|c|c|}
\hline \multirow[t]{2}{*}{$\mathrm{HCC}$ risk factors/TLs } & \multicolumn{2}{|c|}{$\mathrm{N}(\%)$} & \multirow[t]{2}{*}{ Crude OR $(95 \% \mathrm{CI})$} & \multirow[t]{2}{*}{ OR* $(95 \% \mathrm{CI})$} \\
\hline & Cases & Controls & & \\
\hline \multicolumn{5}{|l|}{ HBsAg/TL, N=794 } \\
\hline HBsAg(-)/Longer & $59(22.3)$ & $215(40.6)$ & 1.00 (Reference) & 1.00 (Reference) \\
\hline HBsAg(-)/Shorter & $38(14.3)$ & $179(33.8)$ & $1.08(0.64-1.84)$ & $1.00(0.53-1.86)$ \\
\hline HBsAg(+)/Longer & $65(24.5)$ & $50(9.4)$ & $5.47(3.23-9.25)^{* *}$ & $7.85(4.17-14.80)^{* *}$ \\
\hline HBsAg(+)/Shorter & $103(38.9)$ & $85(16.2)$ & $6.36(3.74-10.81)^{* *}$ & $6.30(3.45-11.51)^{* *}$ \\
\hline RER $(95 \% \mathrm{CI})$ & & & $0.81(-2.11$ to 3.73$)$ & $-1.55(-4.57$ to 1.47$)$ \\
\hline \multicolumn{5}{|l|}{ Anti-HCV/TL, N=703 } \\
\hline Anti-HCV(-)/Shorter & $105(43.8)$ & $221(47.6)$ & 1.00 (Reference) & 1.00 (Reference) \\
\hline Anti-HCV(-)/Longer & $74(31.0)$ & 199 (42.9) & $0.74(0.48-1.12)$ & $0.97(0.59-1.60)$ \\
\hline Anti-HCV $(+) /$ Shorter & $30(12.6)$ & $30(6.5)$ & $2.46(1.30-4.67)^{* *}$ & $4.64(2.13-10.11)^{* *}$ \\
\hline Anti-HCV(+)/Longer & $30(12.6)$ & $14(3.0)$ & $6.51(2.68-15.82)^{* *}$ & $11.47(4.10-32.08)^{* *}$ \\
\hline RER $(95 \%$ CI $)$ & & & $4.32(1.66$ to 6.97$)$ & $6.86(2.34$ to 11.40$)$ \\
\hline \multicolumn{5}{|c|}{ Cigarette smoking/TL, N=800 } \\
\hline Never/Longer & $84(31.3)$ & $181(34.0)$ & 1.00 (Reference) & 1.00 (Reference) \\
\hline Never/Shorter & $79(29.5)$ & $152(28.6)$ & $1.20(0.78-1.84)$ & $0.74(0.419-1.34)$ \\
\hline Ever/Longer & $40(14.9)$ & $85(16.0)$ & $1.06(0.63-1.80)$ & $0.74(0.37-1.46)$ \\
\hline Ever/Shorter & $65(24.3)$ & $114(21.4)$ & $1.37(0.83-2.27)$ & $0.82(0.43-1.59)$ \\
\hline RER $(95 \% \mathrm{CI})$ & & & $0.11(-0.67$ to 0.89$)$ & $0.36(-0.16$ to 0.87$)$ \\
\hline \multicolumn{5}{|c|}{ Alcohol drinking/TL, N=799 } \\
\hline Never/Longer & $110(41.2)$ & $239(44.9)$ & 1.00 (Reference) & 1.00 (Reference) \\
\hline Never/Shorter & $110(41.2)$ & $229(43.0)$ & $1.12(0.77-1.63)$ & $0.81(0.49-1.32)$ \\
\hline Ever/Longer & $14(5.2)$ & $27(5.1)$ & $1.15(0.57-2.33)$ & $1.20(0.50-2.89)$ \\
\hline Ever/Shorter & $33(12.4)$ & $37(7.0)$ & $2.27(1.25-4.13)^{* *}$ & $1.96(0.92-4.19)$ \\
\hline RER $(95 \% \mathrm{CI})$ & & & $1.00(0.13$ to 1.87$)$ & $0.95(0.21$ to 1.69$)$ \\
\hline
\end{tabular}

$*$ OR adjusted for age, gender and other three HCC risk factors except for the factor that has potential modification effect with TL; ** $p<0.01$.

\section{Discussion}

The association between PBL TL and HCC risk was investigated in a prospective cohort recruited in Taiwan. Shorter PBL TL was observed in HCC cases compared with matched controls but the difference was not statistically significant (Table I). Overall, no statistically significant associations between shorter TL and increased HCC risk were observed when categorizing TL by median, tertile or quartile of controls (Table III). The only significant case- 
Table V. Association between time (years) of blood collection prior to HCC diagnosis and peripheral blood leucocyte telomere length

\begin{tabular}{lcccc}
\hline $\begin{array}{l}\text { Blood collection } \\
\text { groups }\end{array}$ & $\begin{array}{c}\text { TL } \\
\text { Median }(\mathrm{IQR}) *\end{array}$ & $\begin{array}{c}\text { Longer TL }(\geq 42.19) \\
(\mathrm{N}, \%)\end{array}$ & $\begin{array}{c}\text { Shorter TL }(<42.19) \\
(\mathrm{N}, \%)\end{array}$ & OR (95\%CI)** \\
\hline$>10$ years & $55.13(46.79)$ & $75(63.0)$ & $44(37.0)$ & $1.00($ Reference $)$ \\
5 - 10 years & $37.09(47.28)$ & $29(45.3)$ & $35(54.7)$ & $2.16(1.10-4.24)$ \\
$<5$ years & $27.62(23.31)$ & $20(23.5)$ & $65(76.5)$ & $3.93(2.00-7.72)$ \\
\hline
\end{tabular}

*ANOVA test $p<0.001$. **OR adjusted for age, gender, smoke, alcohol use, HBV infection and HCV infection using blood collection $>10$ years prior to HCC diagnosis as reference.

control difference was observed among ever alcohol drinkers (Table II), with HCC cases having significantly shorter TL than controls. We found a significant modification effect of carrying longer TL and being anti-HCV positive on increased HCC risk even after adjusting for other risk factors (Table IV). Our study also found a potential interaction effect of shorter TL and ever alcohol drinking on increased HCC risk, although it was not significant after adjusting for other HCC risk factors. Two previous studies showed that longer TL alone was associated with increased HCC risks $(11,12)$ and an interaction between longer TL and ever alcohol use was associated with increased HCC risk (11), contradicting our observations. One study used a case-control study design to measure PBL TL after cancer diagnosis and thus it may have a reverse result (11). The other study measured TL in prospectively collected serum samples (not PBL) (12), which represents a mixture of TLs in a variety of cells that may not be an ideal surrogate to explore cancer risk biomarkers.

Maintaining TL requires balancing between telomere shortening and lengthening processes. Telomere shortening usually occurs during the initiation of cancer and about $85 \%$ of cancers increase their telomerase activity to maintain TL (15). Therefore, both longer and shorter TL may be found associated with increased cancer risk (16), including for $\operatorname{HCC}(8,9)$. DNA from PBLs largely reflects the constitutional genetic background, but chemotherapy after cancer diagnosis may lead to telomere loss. Eight weeks of chemotherapy for head and neck tumors was shown to reduce TL by 660 bps (17). Thus, measuring TL in leukocytes collected prior to cancer development should provide better risk prediction. However, most previous studies showed inconsistent associations between PBL TL and cancer risk (18). This may be due to the fact that samples were collected after cancer diagnosis or at different tumor stages. Using the prospective CSP cohort, we found a higher proportion of patients with shorter TLs in samples collected close to cancer diagnosis (Table V). Longer TL was significantly associated with increased HCC risk in blood collected over 10 years prior to diagnosis, while shorter TL was associated with increasing risk of HCC in blood collected $<5$ years before diagnosis, suggesting different telomere regulatory mechanisms may be involved during the long term development of HCC. Therefore, using pre-diagnostic samples can minimize the potential impact on TL caused by the tumor itself and clinical treatment and reveal the dynamic characteristics of TLs before hepatocarcinogenesis.

We also found that certain HCC risk factors impact PBL TL (Table II). Both HBV and HCV infection were significantly associated with decreased PBL TL in controls. Males and ever smokers also had significantly shorter TL compared with females and never smokers. Shorter TL was observed in ever alcohol drinkers compared with non-drinkers, but was only significant in HCC cases and not in controls. This may indicate a poor capacity to elongate TL in cases, but that controls still have compensatory ability to lengthen TL. It is known that shortened PBL TL is a sign of immunosenescence $(5,8,9)$. Telomerase, regulated by its catalytic subunit (hTERT), also plays a key role in lymphocyte replicative life span. Previous studies found that HBV and HCV infection increased T-lymphocyte apoptosis (19) by inducing P21 and significantly decreasing hTERT mRNA expression and telomerase activity (20). This is consistent with our observation and a previous report that patients with chronic HCV infection had shorter leukocyte TL (12).

Cigarette smoking and alcohol drinking may heighten systemic oxidative stress and inflammation and lead to accelerated telomere shortening. Several previous studies indicated that if telomeric DNA is deficient in repairing single-strand breaks induced by oxidative damage, TL may decrease with increased oxidative stress (21). Many previous studies supported our observation, i.e. an inverse relationship between cigarette smoking and shorter TL $(22,23)$. These results indicate the important biological role of $\mathrm{TL}$ as a biomarker of carcinogenic exposures, which may help better evaluate $\mathrm{HCC}$ risk.

The prospective nested case-control study design of the current study used PBL DNA collected years prior to HCC diagnosis to investigate the role of TL in HCC risk. Our results are thus more likely to reflect the true association because we can exclude the potential impacts of tumor status and treatment. Our study has the largest sample size to date in 
analyzing PBL TL and HCC risk, which ensures sufficient statistical power to analyze the main effect of shorter TL, as well as the potential modification effects of other HCC risk factors. Quantitative PCR has been widely used to evaluate PBL TL and cancer risk because it does not require live cells, it uses small amounts of DNA and has the potential for high throughput. The qPCR method, developed by Cawthon (24) amplifies both telomeric DNA and a single copy gene (albumin) in separate plates; the latest version (25) puts the two reactions into the same well (monochrome multiplex qPCR) to reduce variation of input DNA. But this assay only uses the $\mathrm{T} / \mathrm{S}$ ratio to estimate relative TL making it difficult to compare results between various studies. Based on the original qPCR method, O'Callaghan et al. made a modification by separately assaying two oligomer standards to generate the absolute TL (26). In the current study, we further modified the assay by using both monochrome multiplex qPCR and two oligomer standards within one plate/well to obtain absolute TL. This modification not only increases the accuracy of qPCR, but also makes it easy to compare absolute TLs between studies.

There are two limitations to our study. We assessed absolute TL by a modified qPCR assay, but did not validate the results by the telomeric restriction fragment (TRF) assay that is considered the gold standard method. Secondly, the sample collection years prior to HCC diagnosis varied considerably (from 6 months to 18 years) that may dilute the true relationship if many samples were collected far from HCC diagnosis. Serially collected samples would help to further clarify the real association of PBL TL and HCC risk.

In summary, although shorter PBL TL alone is nonsignificantly associated with increased HCC risk in our nested case-control study using DNA samples collected 6 months to 18 years prior to cancer diagnosis, potential modification effects with anti-HCV status and ever alcohol drinking were observed. $\mathrm{HCC}$ risk factors including $\mathrm{HBV} / \mathrm{HCV}$ infection and ever smoking were also significantly associated with shorter TL in controls. A further large prospective study with serially collected PBL is warranted to clarify the etiologic role of aberrant TL in HCC risk.

\section{Acknowledgements}

This work was financially supported by NIH grants R03 CA156629, R01 ES005116, P30 ES009089 and P30 CA013696. The Authors declare no potential conflicts of interest.

\section{References}

1 Greider CW: Telomere length regulation. Annu Rev Biochem 65: 337-365, 1996.

2 Huffman KE, Levene SD, Tesmer VM, Shay JW and Wright WE: Telomere shortening is proportional to the size of the Grich telomeric 3'-overhang. J Biol Chem 275: 19719-19722, 2000 .
3 Wright WE, Tesmer VM, Huffman KE, Levene SD and Shay JW: Normal human chromosomes have long G-rich telomeric overhangs at one end. Genes Dev 11: 2801-2809, 1997.

4 Artandi SE, Chang S, Lee SL, Alson S, Gottlieb GJ, Chin L and DePinho RA: Telomere dysfunction promotes non-reciprocal translocations and epithelial cancers in mice. Nature 406: 641645, 2000.

5 Maser RS and DePinho RA: Connecting chromosomes, crisis, and cancer. Science 297: 565-569, 2002.

6 Zhang Y, Shen J, Ming W, Lee YP and Santella RM: Telomere length in hepatocellular carcinoma and paired adjacent non-tumor tissues by quantitative PCR. Cancer Invest 25: 668-677, 2007.

7 Satyanarayana A, Manns MP and Rudolph KL: Telomeres and telomerase: a dual role in hepatocarcinogenesis. Hepatology 40: 276-283, 2004.

8 Oh B-K, Jo Chae K, Park C, Kim K, Jung Lee W, Han K-h and Nyun Park Y: Telomere shortening and telomerase reactivation in dysplastic nodules of human hepatocarcinogenesis. J Hepatol 39: 786-792, 2003.

9 Oh B-K, Kim Y-J, Park C and Park YN: Up-regulation of telomere-binding proteins, TRF1, TRF2, and TIN2 is related to telomere shortening during human multistep hepatocarcinogenesis. Am J Pathol 166: 73-80, 2005.

10 Oh BK, Kim H, Park YN, Yoo JE, Choi J, Kim KS, Lee JJ and Park C: High telomerase activity and long telomeres in advanced hepatocellular carcinomas with poor prognosis. Lab Invest 88 : 144-152, 2008.

11 Liu J, Yang Y, Zhang H, Zhao S, Liu H, Ge N, Yang H, Xing JL and Chen $\mathrm{Z}$ : Longer leukocyte telomere length predicts increased risk of hepatitis B virus-related hepatocellular carcinoma: a casecontrol analysis. Cancer 117: 4247-4256, 2011.

12 Fu X, Wan S, Hann HW, Myers RE, Hann RS, Au J, Chen B, Xing $J$ and Yang H: Relative telomere length: a novel noninvasive biomarker for the risk of non-cirrhotic hepatocellular carcinoma in patients with chronic hepatitis B infection. Eur J Cancer 48: 1014-1022, 2012.

13 Wu HC, Wang Q, Wang LW, Yang HI, Ahsan H, Tsai WY, Wang LY, Chen SY, Chen CJ and Santella RM: Urinary 8-oxodeoxyguanosine, aflatoxin B1 exposure and hepatitis B virus infection and hepatocellular carcinoma in Taiwan. Carcinogenesis 28: 995-999, 2007.

14 Andersson T, Alfredsson L, Kallberg H, Zdravkovic S and Ahlbom A: Calculating measures of biological interaction. Eur J Epidemiol 20: 575-579, 2005.

15 Cesare AJ and Reddel RR: Alternative lengthening of telomeres: models, mechanisms and implications. Nat Rev Genet 11: 319$330,2010$.

16 Wentzensen IM, Mirabello L, Pfeiffer RM and Savage SA: The association of telomere length and cancer: a meta-analysis. Cancer Epidemiol Biomarkers Prev 20: 1238-1250, 2011.

17 Unryn BM, Hao D, Gluck S and Riabowol KT: Acceleration of telomere loss by chemotherapy is greater in older patients with locally advanced head and neck cancer. Clin Cancer Res 12: 6345-6350, 2006.

18 Hou L, Zhang X, Gawron AJ and Liu J: Surrogate tissue telomere length and cancer risk: shorter or longer? Cancer Lett 319: 130-135, 2012.

19 Nakamura K, Yuh K, Sugyo S, Shijo H, Kimura N and Okumura M: Apoptosis observed in peripheral T lymphocytes from patients with chronic hepatitis B. Gastroenterology 111: 156-164, 1996. 
20 Zhu CW, Chen M, Luo XR, Wang HY, Wang LH, Wu JH, Li M, Zhang XH, Zhu W, Ye JZ and Qian F: Interferon alpha on expression of hTERT mRNA in peripheral blood mononuclear cells of patients with chronic hepatitis B. Clin Dev Immunol 2011: 920146, 2011.

21 Kawanishi S and Oikawa S: Mechanism of telomere shortening by oxidative stress. Ann NY Acad Sci 1019: 278-284, 2004.

22 Song Z, von Figura G, Liu Y, Kraus JM, Torrice C, Dillon P, Rudolph-Watabe M, Ju Z, Kestler HA, Sanoff H and Lenhard Rudolph K: Lifestyle impacts on the aging-associated expression of biomarkers of DNA damage and telomere dysfunction in human blood. Aging Cell 9: 607-615, 2010.

23 Huzen J, Wong LSM, van Veldhuisen DJ, Samani NJ, Zwinderman AH, Codd V, Cawthon RM, Benus GFJD, van der Horst ICC, Navis G, Bakker SJL, Gansevoort RT, de Jong PE, Hillege HL, van Gilst WH, de Boer RA and van der Harst P: Telomere length loss due to smoking and metabolic traits. J Intern Med 275: 155-163, 2014.
24 Cawthon RM: Telomere measurement by quantitative PCR. Nucleic Acids Res 30: e47, 2002.

25 Cawthon RM: Telomere length measurement by a novel monochrome multiplex quantitative PCR method. Nucleic Acids Res 37: e21, 2009.

26 O'Callaghan N, Dhillon V, Thomas $\mathrm{P}$ and Fenech M: A quantitative real-time PCR method for absolute telomere length. BioTechniques 44: 807-809, 2008.

Received November 10, 2016

Revised December 22, 2016

Accepted December 30, 2016 